\title{
A novel Scheme on the Seamless Handover on Vehicle
}

\author{
YAO Linyuan ${ }^{1, a}$, TANG He ${ }^{2, b}$, DONG Ping ${ }^{1, c}$ and KONG Yong ${ }^{3, d}$ \\ ${ }^{1}$ Beijing Jiaotong University, Beijing, China \\ ${ }^{2}$ Beijing Telecom Planning \& Designing Institute Co. Ltd, Beijing, China \\ ${ }^{3}$ KYLAND Technology Co. Ltd, Beijing, China \\ a,c $\left\{11111020\right.$, pdong\}@bjtu.edu.cn, ${ }^{b}$ tanghe@btpdi.com.cn, ${ }^{d}$ kongyong@kyland.com.cn
}

Keywords: handover, seamless, vehicle, communication.

\begin{abstract}
In traditional Train-Ground Communication (TGC), the mobile node or the mobile network is responsible to handover from one domain to another, although the designer has the route of the trains. A novel scheme on the seamless handover on vehicle is proposed in this paper. The main idea is that the channel would be controlled to adapt the mobile train instead of changing the channel or connection according to the prepared route between the mobile node and the network on the ground. The simulated results show the feasibility of the novel scheme.
\end{abstract}

\section{Introduction}

Recently, as the speed of the train gets faster and faster, reliability and security become the critical problems [1]. When the train moves between different domains, handover will happen. Handover in railway network is mainly based on signals transmitted from base stations (BS) to mobile stations (MS) [2]. In [3], upon the train moves into the intersection, it will start to complete trigging, scanning, selection and implementation.

To solve this problem, a lot of research work is in progress, such as MIPv4 [4], MIPv6 [5] [6], HMIPv6 [7], PMIPv4 [8] and PMIPv6 [9]. These programs reduce the network delay caused by network handover, however, all of them do not fundamentally solve the problem that terminal entering a new area needs to re-detect, re-associate, re-certificate.

In this paper, a novel scheme on the seamless handover on vehicle based on the planned route is proposed. We adjust dynamically the channel to adapt the travelling train, instead of changing the channel or connection between the mobile node and the network on the ground, according to the prepared route.

The rest of this paper is organized as follows. Section II presents our proposed Handover Scheme Based on the Changeable Channel (HSBCC). Section III develops a scenario to prove the scheme. Section IV concludes this paper.

\section{A Handover Scheme Based on the Changeable Channel}

In the area of traditional Train-Ground Communication (TGC), the route of mobile terminal has been planned before the train's setting out. In this section, we propose a noble scheme, called a Handover Scheme Based on the Changeable Channel (HSBCC), to achieve the objective that when the train moves in accordance with the planned route, the network on the ground is responsible to the handover and the terminal or the subnet on the train has no sense of the movement.

HSBCC has three functional entities Network Access Point Controller (NAC), Network Access Point (NA) and Mobile Terminal or Subnet (MTS) as illustrated in Figure 1. NAC is responsible to the query, store and update the Track Table of MTS (TTM), which stores the mapping relationship of the identifier, the state and the track of movement, and the State Table of NA (STN), having the mapping relationship of the identifier of NA and MN, the suite of key management. It also establishes a connection and sends the control message to the $\mathrm{MN}$ or subnet and the state modification message to the access network. NA can be looked as the source of the wireless signal to support the link-layer 
communication. In addition, it sends the state message of itself to NAC or receives that from NAC to update itself state. As the network terminal devices, MTS is responsible to receive, carry out and response to the control message from NAC with the ability of communication.

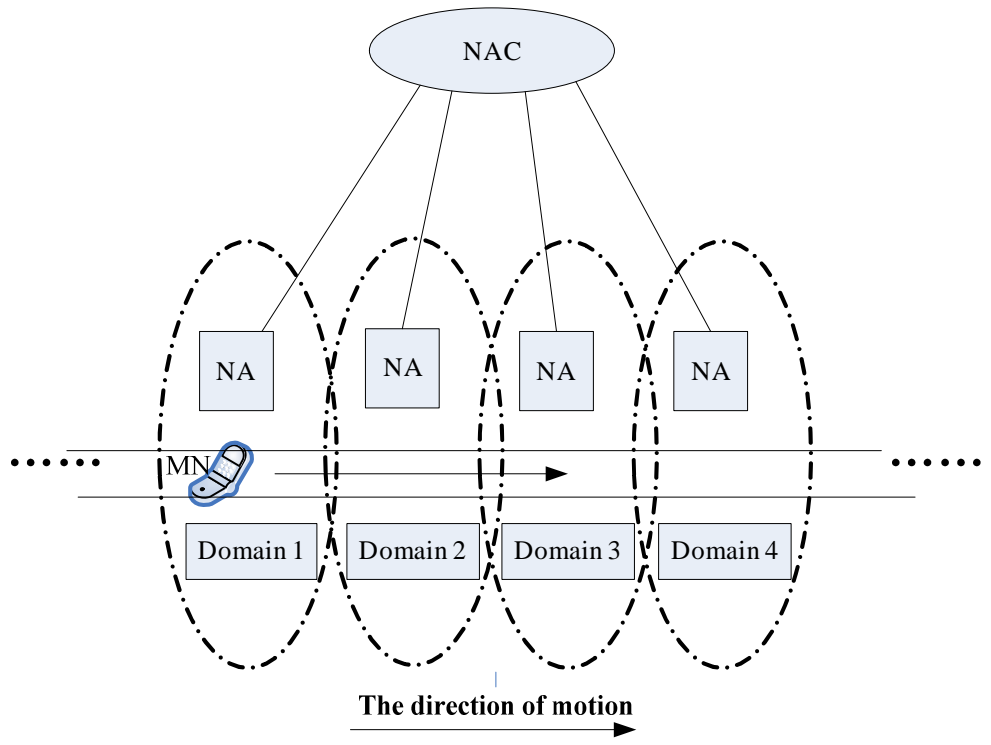

Fig. 1 The model of HSBCC

There are three main processes in this scheme, the process of communication before movement of MTS, the process of handover when moving and the process of communicaiton after movement of MTS. We define the previous NA is the NA having been access to the network, the next NA is NA that is going to access to the network, the past NA is NA that has disconnected to the network.

A. The process of communication before movement of MTS,

Step 1) NAC queries the TTM to select and sends connection request to the MTS;

Step 2) MTS responses to the connection response after getting the request message;

Step 3) MTS updates the TTM and establishes the connection with MTS;

Step 4) NA sends the access message and the state message to NAC;

Step 5) NAC updates STN and transmits state change message to next NA by querying TTM;

Step 6) NA updates itself state message, then sends the success message;

Step 7) NAC changes the STN and transmits the start message to MTS;

Step 8) MTS starts and responses the start message to NAC;

Step 9) NAC updates the TTM.

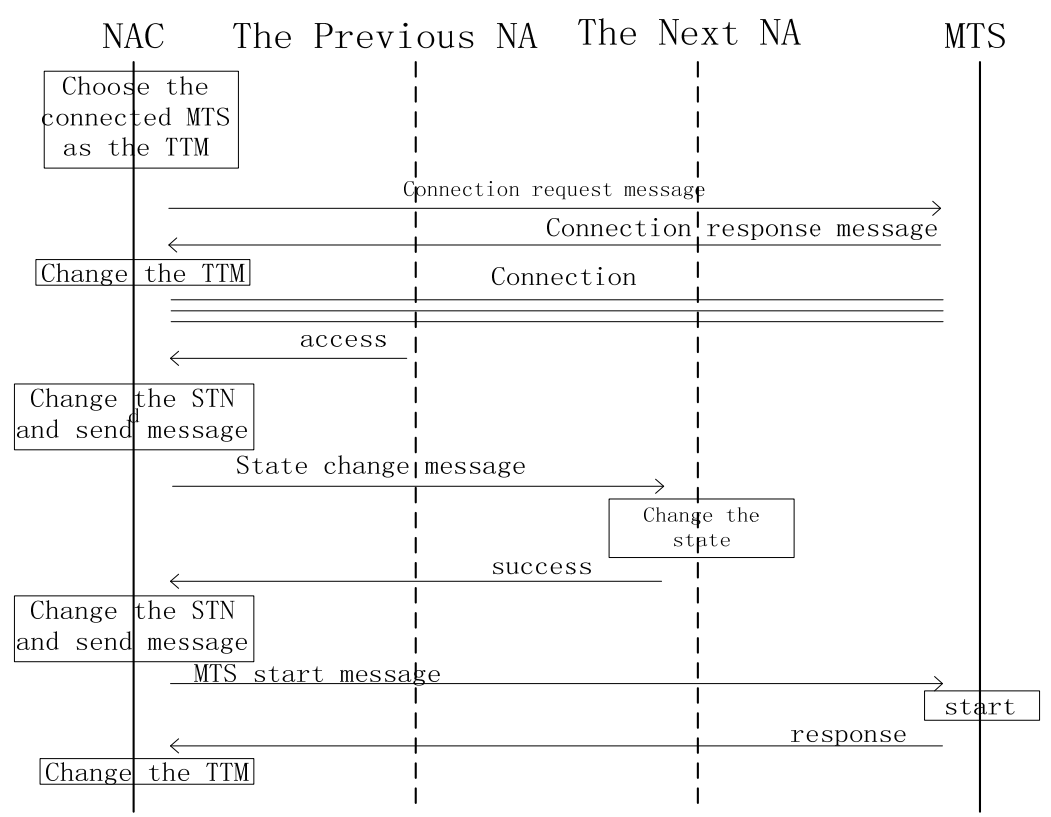

Fig. 2 The process of communication before movement of MTS 
B. The process of handover when moving

Step 1) Now NA transmits the access message to NAC;

Step 2) NAC changes and quarries STN, sends state change message to the previous and next NA;

Step 3) The past NA and the next NA change the state and send the state change message to NAC;

Step 4) NAC updates the STN after receiving the message.

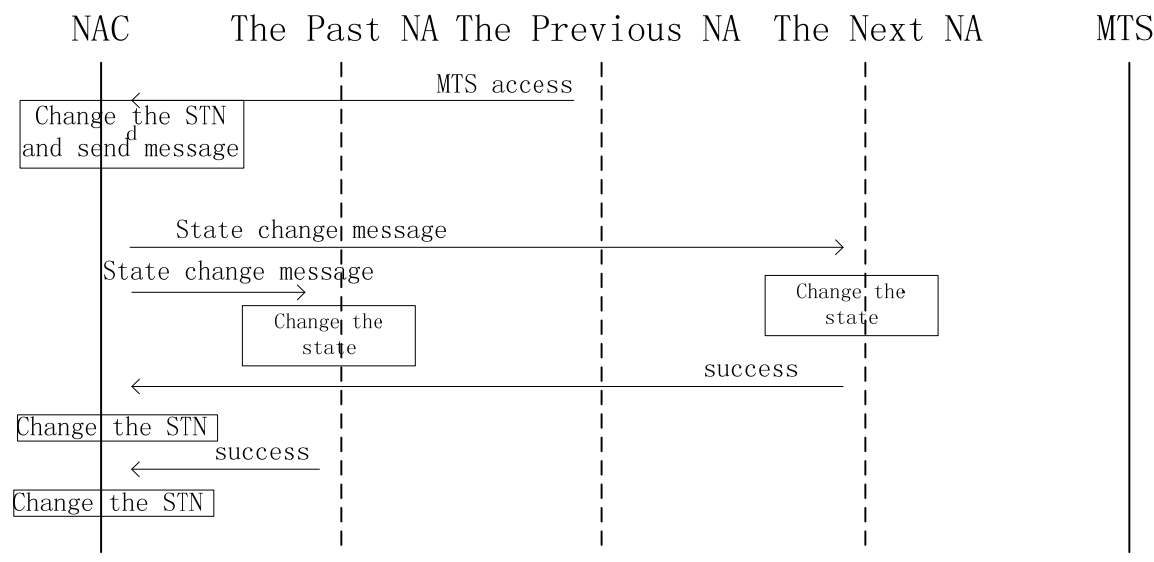

Fig. 3 The process of handover when moving

C. The process of communication after movement

Step 1) The previous NA sends the access message to NAC;

Step 2) NAC queries and updates the TTM and STN, sends the state change message to the past NA and stop message to MTS.

Step 3) The past NA changes the state and responses to NAC;

Step 4) MTS starts the stop process after getting the stop message;

Step 5) Getting state completing message and stop response message, NAC update STN and TTM

Step 6) MTS sends the stop message to NAC when stopping;

Step 7) NAC changes TTM, if getting stop message, sends state change message to previous NA;

Step 8) NAC changes the state and response to the NAC;

Step 9) NAC updates the STN.
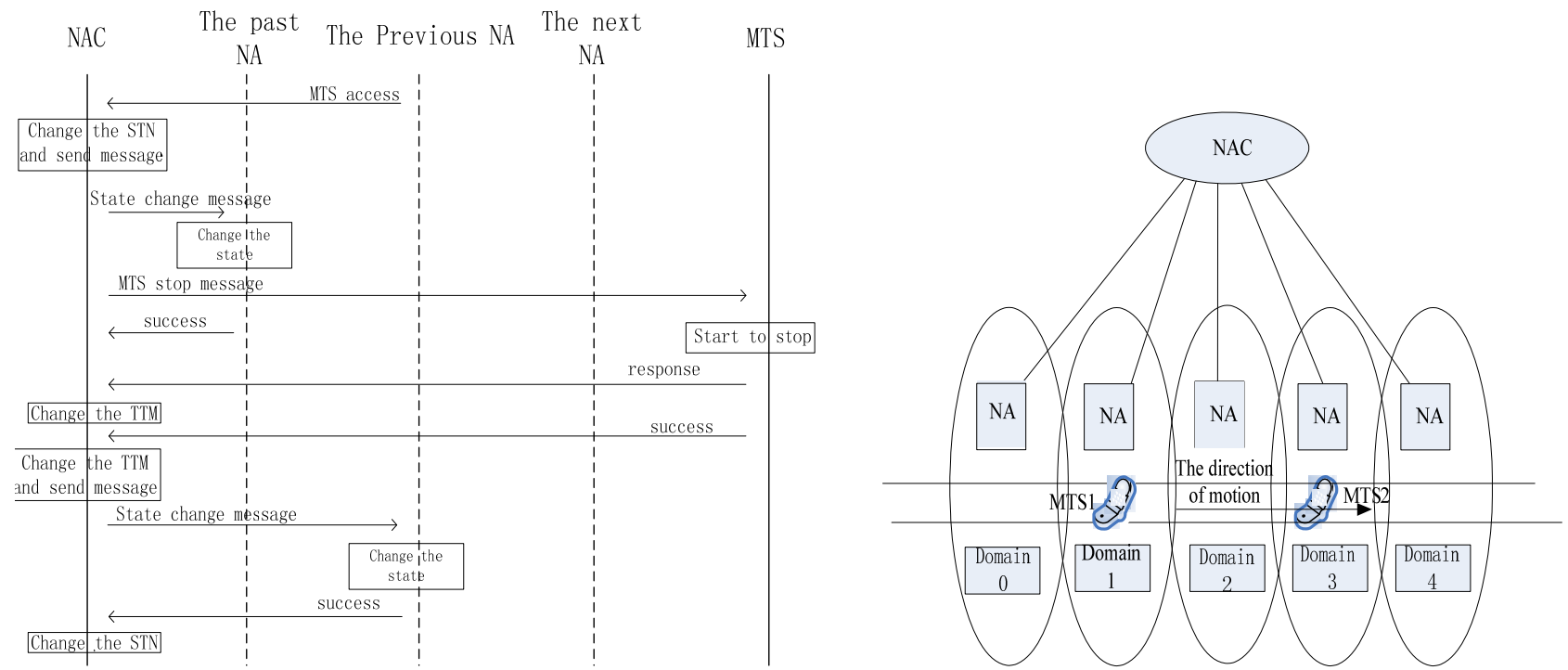

Fig. 4 The process of communication after movement Fig. 5 Two MTS movement on the same track

\section{Analysis of the scenarios}

In this section, a scenario is supposed that there are two MTSs moving on the same track normally, MTS2 in front of MTS1, suddenly, MTS2 has to stop because of unpredictable accident, at that time MTS1 is moving from domain 0 to domain 1. Fig. 5 shows the specific circumstance. 
In the above scenario, the noble scheme could solve this problem to make sure that all the trains are safe. The steps are as follows:

Step 1) When MTS1 moves into domain 1, NA in domain 1 sends the message with MN's moving into domain 1 to NAC;

Step 2) NAC queries the STN and TTM and sends the state change message to NAC;

Step 3) NAs in domain 0 and 2 update state about MTS1, sends the state completion message;

Step 4) NAC updates the STN;

Step 5) when MTS1 moves into domain 2, the NA of domain 2 sends the message to NAC to inform that MTS1 has been in domain 2;

Step 6) NAC queries and updates the TTM and STN and sends the state change message; because of the track being used by MTS2, NAC sends the stop message to MTS1;

Step 7) NA of domain 1 updates the state and sends the completion message. Getting the stop message, MTS starts the stop process;

Step 8) NAC updates the TTM and STN;

Step 9) MTS1 sends the completion message after stopping;

Step 10) NAC updates the TTM, after getting the stop message and prepare to send the start message until detecting the NA of domain 3 free.

\section{Conclusion}

In this paper, a novel scheme on the seamless handover on vehicle is proposed, focusing on the problem of network disruption due to reconnection. The communication connection could be maintained by adapting the network on the ground to the MTS, instead of changing the channel or connection between the mobile node and the network on the ground, according to the prepared route. At last, we suppose a scenario that proves the feasibility of the novel scheme.

\section{References}

[1] Hengliang Tang , Hao Wu, Shiya Wen. A Novel GSM-R Train Control System and Method for Real-time Transmission of Data. Microwave, Antenna, Propagation, and EMC Technologies for Wireless Communications (MAPE), Nov. 2011.

[2] Shiya Wen, Hao Wu, Haoyi Shi. A Novel Handover Mechanism Based on Train Index for GSM-R Network. Microwave, Antenna, Propagation, and EMC Technologies for Wireless Communications (MAPE), Nov. 2011.

[3] Licong Huang, Gang Zhu, ”Analysis and optimization of handover in GSM-R network”, Mobile Communications, 2007,vol.8, pp.35-38.

[4] Jui-Hung Yeh, et al. Fast Intra-Network and Cross-Layer Handover (FINCH) for WiMAX and Mobile Internet, IEEE Transactions on Mobile Computing, Apr 2009.

[5] D. Johnson, C. Perkins, and J. Arkko, “Mobility Support in IPv6,” IETF rfc-3775, June 2004.

[6] R. Koodli, “Mobile IPv6 Fast Handovers,” IETF-rfc 5268, June 2008.

[7] H. Soliman, C. Castelluccia, K. El Malki, and L. Bellier, "Hierarchical Mobile IPv6 Mobility Management (HMIPv6),” IETF rfc-4140, August 2005.

[8] K. Leung and G. Dommety, "WiMAX Forum / 3GPP2 Proxy Mobile IPv4” IETF-rfc 5563, February 2010.

[9] K. Kong and W. Lee, "Mobility Management for All-IP Mobile Networks: Mobile IPv6 vs. Proxy Mobile IPv6,” IEEE Wireless Communications, pp. 36-45, April 2008. 